Kleinhirn auf unzureichender Kenntnis des Verhältnisses des Deiterskerns zum Kleinhirn and der Kleinhirnrinde $\mathrm{zu}$ den Kleinhirnkernen beruhen.

Berlstein kritisiert die galvanische Probe Babin'skis und behauptet, daß diese Reaktion auch nach vollständiger Zerstörung des Vestibularapparates positiv ausfällt, wenn die kalorische und die Drehreaktion bereits negativ sind. Es ist daher zweifelhaft, ob die galvanische Reaktion eine Bedentung für die Diagnose von Erkrankungen des peripheren Vorhofapparates haben kann.

Weitere Zweifel über die galvanische Probe wurden erhoben von Rosenthal und Roth feld, während Jarkowski die gemachten Vorwürfe widerlegt.

Spira-Krakau.

\title{
VI. 17. Internationaler medizinischer Kongreß in London vom 7-12. August 1913. Sektion für Ohrenheilkunde.
}

\author{
Nachtrag über die Fachausstellung.
}

Das anläßlich des Kongresses eröffnete Museum der otologischen Sektion war zweckmäBigerweise im Gebäude des Central technical College untergebracht in unmittelbarer Nachbarschaft des Sitzungssaales. Die mikroskopischen Präparate waren gut sichtbar aufgestellt. Eine große Anzahl von bereitstehenden Mikroskopen machte es auch möglich, reihenweise die ausgestellten mikroskopischen Präparate zu demonstrieren, derart, $\mathrm{da} B$ nach je 24 Stunden die ausgestellten mikroskopischen Objekte ausgewechselt wurden. Jedem der größeren Aussteller war ein Tisch mit zehn Mikroskopen reserviert. Die Sitzungspausen wurden häufig mit mikroskopischen Demonstrationen im Museumsraume ausgefüllt, außerdem war der Samstag Nachmittag (9. August) den mikroskopischen Präparaten vorbehalten.

Auszug aus dem Katalog der Ausstellung.

Urban Pritchard: Schädel des Platypus mit präpariertem Labyrinth. - Modell eines Schnittes durch die Schnecke der Katze.

F. H. Westmacott: Ohrpräparat vom Wal.

Artur Cheatle: Labyrinth der Krähe. - Labyrinth des neugeborenen Menschen. - Mehrere Labyrinthe des erwachsenen Menschen.

H. Secker Walker: Vier Schläfenbeinpräparate von menschlichen Embryonen.

E. D. Davis: Ein Fall von einseitiger kongenitaler MiBbildung des Gehörganges.

J. K. Milne Dickie und J. S. Fraser: Rekonstruktionsmodell des Mittel- und inneren Ohres des Menschen in zwanzigfacher Vergrößerung. Das Modell zeigt 1. das innere Ende des äußeren Gehörganges mit dem. Trommelfell, 2. das Mittelohr mit den Gehörm knöchelchen, Aditus, Antrum, die Luftzellen und das Labyrinth, endlich 3, die knöcherne Labyrinthkapsel mit dem karotischen Kanal, dem Bulbus jugularis, den Aquädukten, dem inneren Gehörgang und dem VII. und VIII. Hirnnerv.

George L. Streeter: Serie von sieben Modellen zur anatomischen Entwicklung des membranösen Labyrinths und des Hörnerven am Menschen.

A. Schönemann (Bern): Rekonstruktionsmodell des menschlichen Schläfenbeins und des Labyrinths. 
G. J. Jenkins: Rekonstruktionsmodell des Gehörorgans eines $16 \mathrm{~mm}$ langen menschlichen Embryos.

A. A. Gray: Serie von anatomischen Präparaten des membranösen Labyrinths bei verschiedenen Vertebraten.

Artur Cheatle: 1. Mißbildung des lateralen knöchernen halbzirkelförmigen Kanals. Rechtes Schläfenbein einer 61jährigen Frau. Der knöcherne äuBere Bogengang wird durch eine einzige große Kavität dargestellt, die sich weit in die Lateralwand des Vestibulum öffnet. Ein kleiner Fortsatz findet sich im hinteren Teile des schiefen Daches der Höhle. Uber den Zustand des membranösen Labyrinths ist nichts bekannt, im übrigen ist das Schläfenbein normal. Im linken Ohre keine Mißbildung. - 2. Drei Fälle von traumatischer Labyrinthfraktur. Erweiterung des inneren Gehörganges dulch ein Fibrom des Hörnerven. - 3. Drei Präparate von Labyrinthtuberkulose. - 4. Fünf Präparate von Karies und Nekrose des Labyrinths. - 5. Nekrose und Exfoliation des gesamten linken Schläfenbeins. - 6. Knöcherne Fixation des Stapes.

W. Kümmel, H. Marx und C. Beck (Heidelberg): 1. Mikroskopische Photogramme (zum Teil autochrom) des menschlichen Ohrlabyrinths. - 2. Labyrintheiterung im Anschinß an Otitis media. - 3. Labyrinthentriundung im AnschluB an Meningitis. - 4. Nichtinfektiöse Veränderungen des menschlichen Ohrlabyrinths. 5. Pathologische Veränderungen am tiexischen Ohrlabyrinth. 6. Zystenbildung einer Crista acustica des Meerschweinchens. 7. Experimentelle Vexänderungen am Meerschweinchen- und Taubenlabyrinth durch Radium. - 8. Veränderungen der Meerschweinchenschnecke nach längerer Einwirkung von Pfeifentönen. -9 . Labyrintheiterung an einem wilden Hasen. -10 . Kongenitale MiBbildung des Ohres. - 11. Mikroskopische Präparate vom experimentell toxisch geschädigten Fazialis und Nervus octavus.

J. S. Fraser: Mikroskopische Präparate. 1. Akute und chronische Mittelohreiterung. - 2. Tumor des Akustikus mit Taubheit (zwei Präparate). - 3. Tuberkulose des Mittelohrs und des Labyrinths. - 4. Traumatische Fraktur des Felsenbeins. - 5. Traumatische Fraktur der Schädelbasis mit Labyrinthfraktur. - 6. Neuritis des VII. und VIII. Hirnnerven. - 7. Senile Taubheit (Färbung mit Eisenhämatoxylin). - 8) Mehrere Fälle von Aknstikustumor. - 9. Zwei Fälle von Taubstummheit.

A. A. Gray: Mikroskopische Präparate, Ganglion spirale einer tauben Katze. - Otosklerose (3 Fälle).

Brühl (Berlin): Mikroskopische Präparate. 20 Präparate über otosklerose und Spongiosierung des Schläfenbeins.

Guys Hospital Museum: Mikroskopische Präparate. Sarkom des Nasopharynx mit Durchwucherung der Basis des Schädels und des Schläfenbeins, Vorwachsen des Tumors in den inneren und den üußeren Grehörgang. $31 / 2$ jähriges Kind.

G. Alexander (Wien): Makroskopische Präparate. 1. Schnitt durch das Gehörorgan mit Cholesteatom des Mittelohrs und des Labyrinths. - 2. Mazeriertes Schläfenbein von Fällen von chronischer Labyrintheiterung mit Karies der Labyrinthkapsel. Labyrinthsequester. - 3. Fxostose einer lateralen Labyrinthwand. 4. Kongenitale Deformitäten des Mittelohrs und des Labyrinths.

Mikroskopische Präparate. 1. Verschiedene Stadien der akuten und chronischen Labyrintheiterung. - 2. Labyrinthitis ossificans Alexander. - 3. Kongenitale Taubheit mit Defekt der sinualen Septa in der Schnecke (Alexander). - 4. Macula,

Archiv i. Ohrenheilkunde, Bd. 97. Otolog. Rundschau. 
neglecta im menschlichen Ohrlabyrinth. - 5. Abnormitäten im histologischen Bau des normalen menschlichen Ohrlabyrinths. 6. Corpora amylacea im Nervus octavus. - 7. Kongenitale Taubheit. - 8. Otosklerose. - 9. Zirkumskripte chronische Labyrintheiterung. - 10. Atrophie des Cortischen Organs im Anschlus an Arteriosklerose. - 11. Angiosarkom des Nervus octavus. 12. Neurofibrom des Acusticus.

Modelle. 1. Modell des knöchernen und des membranösen Labyrinths in Vergxößerung von 20:1 linear. - 2. Serie von 12 Modellen zur embryologischen Entwicklung des membranösen Labyxinths des Meerschweinchens. - 3. Serie von 14 Modellen zur embryologischen Entwicklung des membranösen Labyrinths von Echidna aculeata.

Apparate und Instrumente. 1. Vorrichtung zur Registrierung der Stabilität des menschlichen Körpers (nach Dx. Theimer). 2. Drehstuhl für die klinische Untersuchung des Vestibularapparates. - 3. Instrumente zur operativen Reselktion des Ohrlabyrinths nach Alexander.

H. Secker Walker: Ohrpräparate eines Falles von chronischer Mittelohreiterung mit Sequesterbildung (12jähriges Kind, Tod an KleinhirnabszeB). - Topographisches Ohrpräparat von chronischer Mittelohreiterung mit Sequestration des Labyrinths. - Labyrinthsequester, bestehend aus Schnecke, Anteilen des Vestibulum und der halbzirkelförmigen Kanäle.

C. Ernest West: Präparat eines Schneckensequesters (12jähriger Knabe). - Labyrinthsequester operativ entfernt.

F. H. West macott: Exfoliation des knöchernen Labyrinths an einem 4jährigen Kinde.

St. Mary's Hospital Museum: Sequester, umfassend das knöcherne Labyrinth, den inneren Gehörgang und die Spitze des Felsenbeins an einem Mädchen mit kongenitaler Lues, durch Ausspritzen entfernt.

Addenbroke's Hospital Museum (Cambridge): Linkes Schläfenbein mit großer Knochenhöhle infolge von Karies im oberen und hinteren Teile des Felsenbeins. Labyrinth vollkommen durch die Karies zerstört. Der innere Gehörgang mündet in die Knochenhöhle.

Hugh E. Jones: Sequester, der fast das ganze Labyrinth umfaht. Präparat von einem Falle von chronischer Mittelohreiterung an einem 21jährigen Mädchen mit kongenitaler Lues, Keine anamnestischen Angaben von Schwindel.

Wyatt Wingrave (Pathologist, Central London Throat and Ear Hospital, London): Chronische Mittelohreiterung mit spontaner Ausstoßung eines das gesamte Labyrinth umfassenden Sequesters, 6 Monate nach der Radikaloperation. - Linkes Schläfenbein mit akuter Labyrintheiterung und Meningitis nach chronischer Mittelohreiterung.

L. B. Rawling: Drei Präparate von Schädelbasisfralktur mit Verletzung des Labyrinths.

Ricardo Botey (Barcelona): Serie von Knochenpräparaten mit Darstellung der versehiedenen Methoden der operativen Eröffnung und Entfermung des Labyrinths.

Hugh E. Jones: Präparat zur Illustration der Methode der Labyrintheröffnung und Zerstörung der vestibularen Nervenendstellen ohne Eröffnung des Mittelohrs. Anzuwenden für Fälle von Schwindel bei nicht eitrigen Ohrerkrankungen. 
C. Ernest West: Präparatenreihe zur Illustration der oberen, doppelten und unteren Vestibulotomie und der translabyrinthären Dränage der basalen Meningitis.

Boenninghaus (Breslau): Vier Modelle zur Anatomie des inneren Ohres. - Schädelbasis mit Bruch des Felsenbeins und des inneren Gehörganges. - Schädelbasisfraktur mit Bruch des Felsenbeins und Eröffnung der halbzirkelförmigen Kanäle und der Eustachischen Röhre und Zerreißung des Trommelfells.

Alexander-Wien. 